\title{
The annual gas demand forecasting in air-source gas-fired absorption heat pumps
}

\author{
Piotr Langowski ${ }^{1}$, Jakub Doroszkiewicz ${ }^{1}$, and Piotr Jadwiszczak ${ }^{2, *}$ \\ ${ }^{1}$ GAZUNO, Al. Zwycięstwa 96/98, 81-451 Gdynia, Poland \\ ${ }^{2}$ Wroclaw University of Science and Technology, Faculty of Environmental Engineering, \\ Plac Grunwaldzki 13, 50-377 Wrocław, Poland
}

\begin{abstract}
The forecasting of fuel demand is required for decision-making and management of heating systems. A simple methodology of gas demand forecasting in heating systems based on air-source gas-fired absorption heat pumps (GAHP) was presented and evaluated on real gas consumption data. The methodology was developed by Gazuno, based on experience and longterm measurements in real GAHP heating systems in Polish climate conditions. Methodology takes into account main factors influencing the gas consumption: the variability of heat pumps' gas utilization efficiency (GUE), the operation conditions and the operation time. In air-source gasfired heat pump technology the GUE changes in operation time according to the external air temperature and heating system temperature. The presented simple methodology is dedicated for decision-making on early design stage, to simple assessment of future gas demand in the GAHP heating systems.
\end{abstract}

\section{Introduction}

The selection of heat source technology is one of the most long-term decision need to be made in every design of new, modernized or replaced heating system. The fuel demand is the important criterion in selecting an appropriate technology to supply the space heating and domestic hot water (DHW). At the early decision-making stage, a simple and powerful tool is needed to provide accurate forecasts of future gas consumption, without the need for extensive models of building and heating systems, like in simulation methods $[1,2]$.

Nowadays the condensing heating boilers have become the common technology in the built environment. The gas-fired absorption heat pumps (GAHP) are described as the next step in efficiency and environment improvement. The GAHP technology combines the advantages of two heating technologies as condensing heating boiler and air-source heat pump. The air-source GAHP can be defined as a high efficiency air-cooled heat pump powered directly by gas as a gas boiler. The GAHP are treated as renewable in European Union with A++ rating and have lower carbon emissions than the gas boiler. The air-source gas-fired absorption heat pumps, as a renewable heating solution, use natural gas or LPG at a high efficiency to converting energy from the surrounding air into high grade heat for space heating and domestic hot water (DHW). Manufacturers of GAHP advertise efficiencies up to $165 \%$, compared to condensing boilers with $110 \%$. The efficiency of GAHP is measured by

\footnotetext{
* Corresponding author: piotr.jadwiszczak@pwr.edu.pl
} 
its gas utilisation efficiency (GUE), as a ratio of the energy supplied by GAHP to the energy consumed in gas [3-8].

\section{GAHP operation characteristic}

Air-source gas-fired absorption heat pumps use gas as a driving energy and external air as a heat source. In all air-source heat pump technologies the efficiency and thermal capacity fall with the decrease of external air temperature and with increase of heating system temperature. These variability must be taken into account in technical design and in gas demand forecasting in GAHP heating systems. Figure 1 presents the GUE for two air-source GAHP types delivered by Gazuno company: K-18 and GAHP-A [3, 4]. Figure 2 shows the thermal capacity of K-18 and GAHP-A units [3, 4].
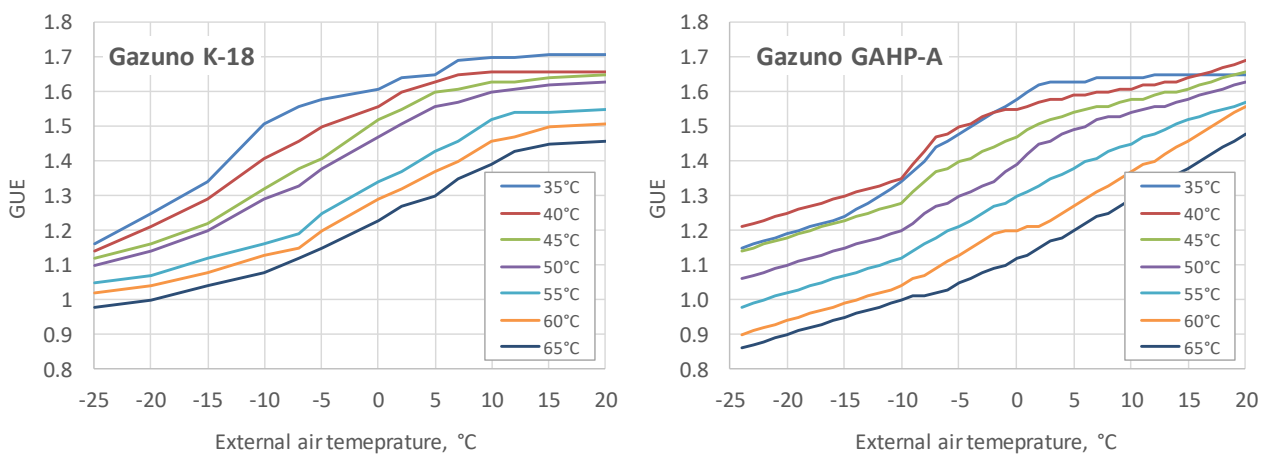

Fig. 1. The gas utilisation efficiency (GUE) of Gazuno K-18 and GAHP-A heat pumps for different heating system temperatures (supply water temperature).
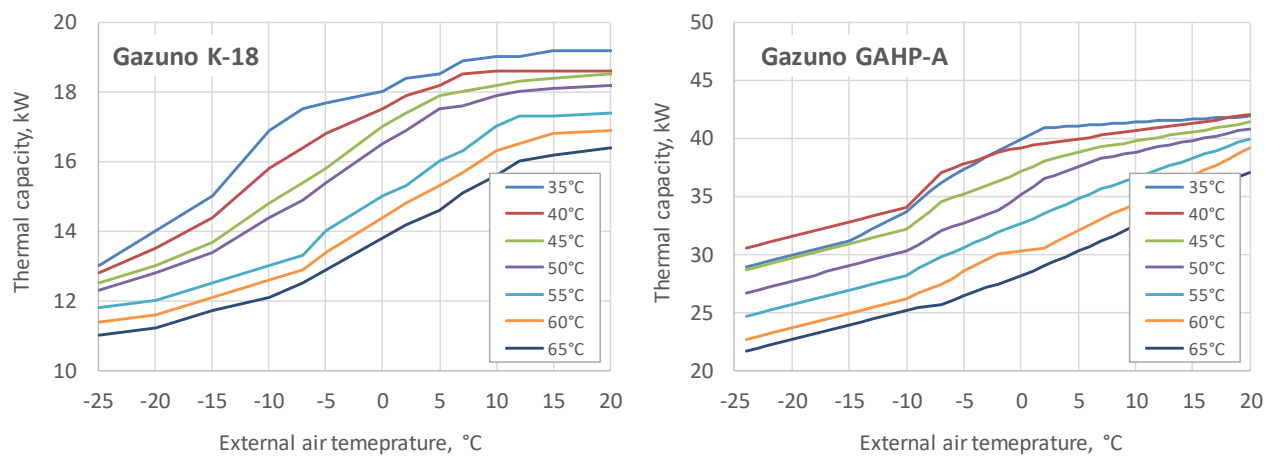

Fig. 2. The thermal capacity of Gazuno K-18 and GAHP-A heat pumps for different heating system temperatures (supply water temperature).

The variability of air-source GAHP operating conditions requires a detailed approach to the fuel demand forecasting. The GAHP manufacturer Gazuno has developed a simple method of annual gas demand forecasting in heating systems based on air-source gas-fired absorption heat pumps in Polish climate conditions. It allows to assess the future fuel demand in the GAHP systems and is dedicated for decision-making support at the early design stage. 


\section{Methodology of annual gas demand forecasting}

Based on technical experience and long-term measurements the simple methodology of gas demand forecasting in GAHP heating systems was developed by Gazuno. The methodology takes into account main factors influencing the gas consumption: the GUE (fig. 1) and GAHP thermal capacity (fig. 2) variability, the connection mode and the operation time of gas-fired heat pumps. The connection modes of GAHP include single source mode, multi-source GAHP cascade mode and combined with condensing gas boiler mode. In the last case the available thermal capacity of GAHP defines the share of heat pumps and boilers in the heat load covering.

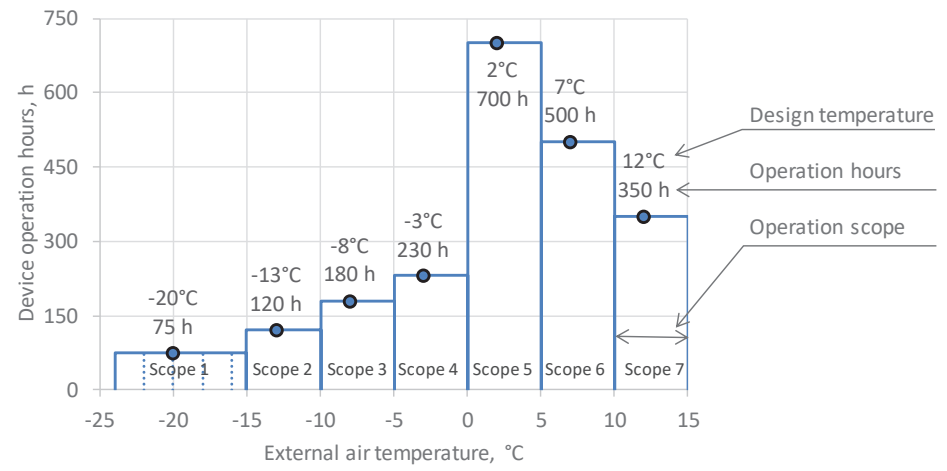

Fig. 3. The seven operation scopes in heating season. Scope 1 differs according to geographical zone.

In order to take into account variable GAHP operation conditions in Gazunos' methodology the heating season in Polish climate was divided into seven operation scopes by external air temperatures $5 \mathrm{~K}$ ranges. The 8 th scope includes the DHW preparation load in summer season. For each operation scope Gazuno has defined the average GAHP operation hours $t_{o, s}$. Figure 3 and table 1 show the defined operation scopes. In first operation scope the external air temperature range and design temperature $\mathrm{T}_{\mathrm{e}, \mathrm{s}}$ change according to the geographic zone.

Table 1. The external air temperature range, the design external air temperature of and the GAHP operation hours of Gazuno operation scopes.

\begin{tabular}{|c|c|c|c|}
\hline $\begin{array}{c}\text { Operation } \\
\text { scope } \\
\text { number }\end{array}$ & $\begin{array}{c}\text { Range of external air } \\
\text { temperature in operation } \\
\text { scope, }{ }^{\circ} \mathrm{C}\end{array}$ & $\begin{array}{c}\text { Design air temperature in } \\
\text { operation scope, } \\
\mathrm{T}_{\mathrm{e}, \mathrm{s}}{ }^{\circ} \mathrm{C}\end{array}$ & $\begin{array}{c}\text { Device operation hours in } \\
\text { operation scope, } \\
\mathrm{t}_{\mathrm{o}, \mathrm{s}}, \mathrm{h}\end{array}$ \\
\hline 1 & $\left(\mathrm{~T}_{\mathrm{e}}{ }^{1)},-15>\right.$ & $-20 \ldots-16^{2)}$ & 75 \\
\hline 2 & $(-15,-10>$ & -13 & 120 \\
\hline 3 & $(-10,-5>$ & -8 & 180 \\
\hline 4 & $(-5,0>$ & -3 & 230 \\
\hline 5 & $(0,5>$ & 2 & 500 \\
\hline 6 & $(5,10>$ & 7 & 350 \\
\hline 7 & $(10,15>$ & 12 & 600 \\
\hline \multicolumn{4}{|c|}{ Domestic hot water preparation in summer mode } \\
\hline 8 & - & 20 & 0 \\
\hline
\end{tabular}


For forecasting of gas demand for space heating the partial heating loads $\mathrm{Q}_{\mathrm{h}, \mathrm{s}}$ for every operation scope need to be calculated based on geographic zone design external temperature $T_{e}$, the scope design air temperature $T_{e, s}$ and internal air design temperature $T_{i}$ :

$$
Q_{h, s}=Q_{h} \cdot \frac{T_{i}-T_{e, s}}{T_{i}-T_{e}}, k W
$$

The heat load can be covered by monovalent GAHP system or by multivalent GAHP and gas heating boiler (HB) system. In multivalent layout the heat pumps are used as base heat source, and HBs support GAHP only in high load periods. The number of GAHP and HB units are determined according to design heat load and annual heat demand profile.

Next the available thermal capacity $\mathrm{Q}_{\mathrm{GAHP}, \mathrm{s}}$ of GAHP units and $\mathrm{GUE}_{\mathrm{s}}$ of GAHP need to be specified for all operational scope conditions. The technical data sheet of GAHP unit includes the GUE and thermal capacity for different external air and heating system temperatures (fig. 1 and 2). In the same way the HB efficiency $\eta$ and thermal capacity $Q_{\mathrm{HB}, \mathrm{s}}$ should be specified for all operation scopes. In every scope the available thermal capacity of heat pumps $\mathrm{Q}_{\mathrm{GAHP}, \mathrm{s}}$ should be compared to scope partial thermal load $\mathrm{Q}_{\mathrm{h}, \mathrm{s}}$. In case $\mathrm{Q}_{\mathrm{h}, \mathrm{s}}>\mathrm{Q}_{\mathrm{GAHP}, \mathrm{s}}$ the HB thermal capacity $\mathrm{Q}_{\mathrm{HB}, \mathrm{s}}$ completes the scope heat load.

The annual gas demand $G$ is a sum of annual gas consumption $G_{h}$ for heating and annual gas consumption $\mathrm{G}_{\mathrm{w}}$ for domestic hot water preparation:

$$
G=G_{h}+G_{w}, m^{3}
$$

The annual gas demand for heating includes the heat pumps' gas consumption $\mathrm{G}_{\mathrm{GAHP}, \mathrm{h}, \mathrm{s}}$ and boilers' gas consumption $\mathrm{G}_{\mathrm{HB}, \mathrm{h}, \mathrm{s}}$ during all heating season operation scopes:

$$
G_{h}=\sum_{s=1}^{7} G_{h, s}=\sum_{s=1}^{7}\left(G_{G A H P, h, s}+G_{H B, h, s}\right)=\sum_{s=1}^{7}\left(\frac{Q_{G A H P, h, s} \cdot t_{o, s}}{G U E_{h, s} \cdot C V_{s}}+\frac{Q_{H B, h, s} \cdot t_{o, s}}{\eta_{s} \cdot C V_{s}}\right), m^{3}
$$

The equation contains the space heating partial load covered by heat pumps $\mathrm{Q}_{\mathrm{GAHP}, \mathrm{h}, \mathrm{s}}$ in $\mathrm{kW}, \mathrm{GUE}_{\mathrm{h}, \mathrm{s}}$, the space heating partial load covered by boilers $\mathrm{QHB}_{\mathrm{h}, \mathrm{s}}$ in $\mathrm{kW}$, heating boiler efficiency $\eta$ in $\%$, device operation time $t_{o, s}$ in hours and calorific value of gas $C_{\mathrm{s}}$ in $\mathrm{kWh} / \mathrm{m}^{3}$ for every operational scope.

The annual gas demand for domestic hot water preparation includes the heat pumps' gas consumption $\mathrm{G}_{\mathrm{GAHP}, \mathrm{w}, \mathrm{s}}$ and boilers' gas consumption $\mathrm{G}_{\mathrm{HB}, \mathrm{w}, \mathrm{s}}$ in eight operation scopes:

$$
G_{w}=\sum_{s=1}^{8} G_{w, s}=\sum_{s=1}^{8}\left(G_{G A H P, w, s}+G_{H B, w, s}\right)=\sum_{s=1}^{8}\left(\frac{Q_{G A H P, w, s} \cdot t_{o, s}}{G U E_{w, s} \cdot C V_{s}}+\frac{Q_{H B, w, s} \cdot t_{o, s}}{\eta_{s} \cdot C V_{s}}\right), m^{3}
$$

The equation contains the DHW average heat load covered by heat pumps $\mathrm{Q}_{\mathrm{GAHP}, \mathrm{w}, \mathrm{s}}$ in $\mathrm{kW}, \mathrm{GUE}_{\mathrm{w}, \mathrm{s}}$, the DHW average heat load covered by boilers $\mathrm{Q}_{\mathrm{HB}, \mathrm{w}, \mathrm{s}}$ in $\mathrm{kW}$, heating boiler efficiency $\eta$ in $\%$, device operation time $t_{o, s}$ in hours and calorific value of gas $\mathrm{CV}_{\mathrm{s}}$ in $\mathrm{kWh} / \mathrm{m}^{3}$ for every operational scope. 


\section{Gas demand forecasting assessment}

To examine and assess the Gazuno methodology the gas demand forecast was calculated and compared to real gas consumption data for three cases, including the Family Support Center, Primary School and Single-family house.

Table 2 shows the input data needed for gas demand forecast calculations. The Family Support Center is supplied by mono-source system based on four air-source gas-fired Gazuno heat pumps GAHP-A. Heat pumps cover the space heating and DHW heat load. In Primary School four GAHP-A units were used supporting by two boilers and solar collectors for DHW. In summer season whole DHW heat load is covered by solar system. The singlefamily house heat load is covered by K-18 Gazuno heat pump supported by existing gas heating boiler.

Table 2. The input data for annual gas demand calculation in analysed cases.

\begin{tabular}{|l|c|c|c|}
\hline \multicolumn{1}{|c|}{ Parameter } & $\begin{array}{c}\text { Family } \\
\text { Support } \\
\text { Center }\end{array}$ & $\begin{array}{c}\text { Primary } \\
\text { School }\end{array}$ & $\begin{array}{c}\text { Single- } \\
\text { family house }\end{array}$ \\
\hline Design external air temperature, ${ }^{\circ} \mathrm{C}$ & -22 & -16 & -16 \\
\hline Design heat load for space heating, $\mathrm{Q}_{\mathrm{h}}, \mathrm{kW}$ & 100 & 161 & 22 \\
\hline Design heat load for DHW, $\mathrm{Q}_{\mathrm{w}}, \mathrm{kW}$ & 22 & $5{ }^{1)}$ & 5.5 \\
\hline Number of heat pump units & $4 \times$ GAHP-A & $4 \times$ GAHP-A & $1 \times \mathrm{K}-18$ \\
\hline Number of heat boilers & - & $2 \times \mathrm{HB}$ & $1 \times \mathrm{HB}$ \\
\hline Natural gas calorific value, $\mathrm{CV}, \mathrm{kWh} / \mathrm{m}^{3}$ & 10.5 & 10.5 & 10.5 \\
\hline
\end{tabular}

Gas demand calculation and input data for three tested cases are shown in tables below: tables 3 and 4 for Family Support Center, tables 5 and 6 for Primary School, tables 7 and 8 for single-family house. In each case the gas demand was forecasted for space heating and DHW needs. The data includes heat demand profile, GAHP and HB connection mode, operation conditions (external air and heating system temperature), GUE and $\eta$ and gas demand in every operation scope.

Table 3. The forecast of annual gas demand for space heating in Family Support Center.

\begin{tabular}{|c|c|c|c|c|c|c|c|c|c|}
\hline Scope & $\begin{array}{c}\text { Partial } \\
\text { heat load } \\
\mathrm{Q}_{\mathrm{h}, \mathrm{s}}, \mathrm{kW}\end{array}$ & $\begin{array}{l}\text { Heating } \\
\text { system } \\
\text { tempera- } \\
\text { ture, }{ }^{\circ} \mathrm{C}\end{array}$ & $\begin{array}{c}\text { Operation } \\
\text { hours, } \\
\mathrm{t}_{\mathrm{o}, \mathrm{s}}, \mathrm{h}\end{array}$ & $\begin{array}{c}\text { Available } \\
\text { GAHP } \\
\text { th.capacity } \\
\text { Q } \\
\text { kAHP,h,s, } \\
\text { kW }\end{array}$ & $\begin{array}{c}\text { GAHP } \\
\text { GUE }_{\mathrm{h}, \mathrm{s}} \\
\text { in heating } \\
\text { mode }\end{array}$ & $\begin{array}{c}\text { GAHP gas } \\
\text { demand } \\
\mathrm{G}_{\mathrm{GAHP}, \mathrm{h}, \mathrm{s}} \\
\mathrm{m}^{3}\end{array}$ & $\begin{array}{c}\mathrm{HB} \\
\text { thermal } \\
\text { capacity, } \\
\text { QHB,h,s, } \\
\text { kW }\end{array}$ & $\begin{array}{c}\mathrm{HB} \text { gas } \\
\text { demand } \\
\mathrm{G}_{\mathrm{HB}, \mathrm{h}, \mathrm{s}}, \\
\mathrm{m}^{3}\end{array}$ & $\begin{array}{c}\text { Gas } \\
\text { demand, } \\
\mathrm{G}_{\mathrm{h}, \mathrm{s}}, \mathrm{m}^{3}\end{array}$ \\
\hline 1 & 93 & 55 & 75 & 103.8 & 1.03 & 644 & 0 & 0 & 644 \\
\hline 2 & 79 & 50 & 120 & 117.9 & 1.17 & 767 & 0 & 0 & 767 \\
\hline 3 & 67 & 45 & 180 & 134.9 & 1.34 & 853 & 0 & 0 & 853 \\
\hline 4 & 55 & 40 & 230 & 153.8 & 1.53 & 784 & 0 & 0 & 784 \\
\hline 5 & 43 & 40 & 700 & 158.2 & 1.57 & 1820 & 0 & 0 & 1820 \\
\hline 6 & 31 & 35 & 500 & 164.9 & 1.64 & 899 & 0 & 0 & 899 \\
\hline 7 & 19 & 35 & 350 & 166.0 & 1.65 & 385 & 0 & 0 & 385 \\
\hline
\end{tabular}


Table 4. The forecast of annual gas demand for domestic hot water in Family Support Center.

\begin{tabular}{|c|c|c|c|c|c|c|c|c|c|}
\hline Scope & $\begin{array}{c}\text { DHW } \\
\text { heat load } \\
\mathrm{Q}_{\mathrm{w}, \mathrm{s}}, \mathrm{kW}\end{array}$ & $\begin{array}{c}\text { DHW } \\
\text { system } \\
\text { tempera- } \\
\text { ture, }{ }^{\circ} \mathrm{C}\end{array}$ & $\begin{array}{c}\text { Operation } \\
\text { hours, } \\
\mathrm{t}_{\mathrm{o}, \mathrm{s}}, \mathrm{h}\end{array}$ & $\begin{array}{c}\text { Available } \\
\text { GAHP } \\
\text { th.capacity } \\
\text { Q }_{\text {GAHP,w,s, }} \\
\text { kW }\end{array}$ & $\begin{array}{c}\text { GAHP } \\
\text { GUE }_{\mathrm{w}, \mathrm{s}} \\
\text { in DHW } \\
\text { mode }\end{array}$ & $\begin{array}{c}\text { GAHP gas } \\
\text { demand } \\
\mathrm{G}_{\mathrm{GAHP}, \mathrm{w}, \mathrm{s}} \\
\mathrm{m}^{3}\end{array}$ & $\begin{array}{c}\mathrm{HB} \\
\text { thermal } \\
\text { capacity, } \\
\text { Q } \\
\text { kW, w,s, } \\
\text { kW }\end{array}$ & $\begin{array}{c}\mathrm{HB} \text { gas } \\
\text { demand } \\
\mathrm{G}_{\mathrm{HB}, \mathrm{w}, \mathrm{s}}, \\
\mathrm{m}^{3}\end{array}$ & $\begin{array}{c}\text { Gas } \\
\text { demand, } \\
\mathrm{G}_{\mathrm{w}, \mathrm{s}}, \mathrm{m}^{3}\end{array}$ \\
\hline 1 & 22 & 55 & 75 & 103.8 & 1.03 & 153 & 0 & 0 & 153 \\
\hline 2 & 22 & 55 & 120 & 109.9 & 1.09 & 231 & 0 & 0 & 231 \\
\hline 3 & 22 & 55 & 180 & 116.9 & 1.16 & 325 & 0 & 0 & 325 \\
\hline 4 & 22 & 55 & 230 & 125.8 & 1.25 & 386 & 0 & 0 & 386 \\
\hline 5 & 22 & 55 & 700 & 134.1 & 1.33 & 1103 & 0 & 0 & 1103 \\
\hline 6 & 22 & 55 & 500 & 142.6 & 1.41 & 743 & 0 & 0 & 743 \\
\hline 7 & 22 & 55 & 350 & 149.2 & 1.48 & 495 & 0 & 0 & 495 \\
\hline 8 & 22 & 55 & 600 & 159.8 & 1.57 & 801 & 0 & 0 & 801 \\
\hline \multicolumn{10}{|c|}{ Annual gas demand for domestic hot water: $G_{w}=4236 \mathrm{~m}^{3} / \mathrm{a}$} \\
\hline
\end{tabular}

Table 5. The forecast of annual gas demand for space heating in Primary School.

\begin{tabular}{|c|c|c|c|c|c|c|c|c|c|}
\hline Scope & $\begin{array}{c}\text { Partial } \\
\text { heat load } \\
\mathrm{Q}_{\mathrm{h}, \mathrm{s}}, \mathrm{kW}\end{array}$ & $\begin{array}{l}\text { Heating } \\
\text { system } \\
\text { tempera- } \\
\text { ture, }{ }^{\circ} \mathrm{C}\end{array}$ & $\begin{array}{c}\text { Operation } \\
\text { hours, } \\
\mathrm{t}_{\mathrm{o}, \mathrm{s}}, \mathrm{h}\end{array}$ & \begin{tabular}{|c|} 
Available \\
GAHP \\
th.capacity \\
Q $_{\text {GAHP,h,s, }}$ \\
kW
\end{tabular} & $\begin{array}{c}\text { GAHP } \\
\text { GUE }_{\mathrm{h}, \mathrm{s}} \\
\text { in heating } \\
\text { mode }\end{array}$ & $\begin{array}{c}\text { GAHP gas } \\
\text { demand } \\
\text { G }_{\mathrm{GAHP}, \mathrm{h}, \mathrm{s}} \\
\mathrm{m}^{3}\end{array}$ & $\begin{array}{c}\mathrm{HB} \\
\text { thermal } \\
\text { capacity, } \\
\mathrm{Q}_{\mathrm{HB}, \mathrm{h}, \mathrm{s}}, \\
\mathrm{kW}\end{array}$ & $\begin{array}{c}\text { HB gas } \\
\text { demand } \\
\mathrm{G}_{\mathrm{HB}, \mathrm{h}, \mathrm{s}}, \\
\mathrm{m}^{3}\end{array}$ & $\begin{array}{c}\text { Gas } \\
\text { demand, } \\
\mathrm{G}_{\mathrm{h}, \mathrm{s}}, \mathrm{m}^{3}\end{array}$ \\
\hline 1 & 161 & 50 & 75 & 114.9 & 1.14 & 1009 & 46 & 336 & 1345 \\
\hline 2 & 148 & 50 & 120 & 117.9 & 1.17 & 1442 & 30 & 346 & 1788 \\
\hline 3 & 125 & 45 & 180 & 134.9 & 1.34 & 1602 & 0 & 0 & 1602 \\
\hline 4 & 103 & 40 & 230 & 153.8 & 1.53 & 1473 & 0 & 0 & 1473 \\
\hline 5 & 81 & 40 & 700 & 158.2 & 1.57 & 3418 & 0 & 0 & 3418 \\
\hline 6 & 58 & 35 & 500 & 164.9 & 1.64 & 1688 & 0 & 0 & 1688 \\
\hline 7 & 36 & 35 & 350 & 166.0 & 1.65 & 723 & 0 & 0 & 723 \\
\hline
\end{tabular}

Annual gas demand for heating: $G_{h}=12036 \mathrm{~m}^{3} / \mathrm{a}$

Table 6. The forecast of annual gas demand for domestic hot water in Primary School.

\begin{tabular}{|c|c|c|c|c|c|c|c|c|c|}
\hline Scope & $\begin{array}{c}\text { DHW } \\
\text { heat load } \\
\mathrm{Q}_{\mathrm{w}, \mathrm{s}}, \mathrm{kW}\end{array}$ & $\begin{array}{c}\text { DHW } \\
\text { system } \\
\text { tempera- } \\
\text { ture, }{ }^{\circ} \mathrm{C}\end{array}$ & $\begin{array}{c}\text { Operation } \\
\text { hours, } \\
\mathrm{t}_{0, \mathrm{~s}}, \mathrm{~h}\end{array}$ & \begin{tabular}{|c|} 
Available \\
GAHP \\
th.capacity \\
Q QAAP,w,s, \\
kW
\end{tabular} & $\begin{array}{c}\text { GAHP } \\
\text { GUE }_{\mathrm{w}, \mathrm{s}} \\
\text { in DHW } \\
\text { mode }\end{array}$ & $\begin{array}{c}\text { GAHP gas } \\
\text { demand } \\
\mathrm{G}_{\mathrm{GAHP}, \mathrm{w}, \mathrm{s}} \\
\mathrm{m}^{3}\end{array}$ & $\begin{array}{c}\mathrm{HB} \\
\text { thermal } \\
\text { capacity, } \\
\text { QHB,w,s, } \\
\mathrm{kW}\end{array}$ & $\begin{array}{c}\mathrm{HB} \text { gas } \\
\text { demand } \\
\mathrm{G}_{\mathrm{HB}, \mathrm{w}, \mathrm{s}} \\
\mathrm{m}^{3}\end{array}$ & $\begin{array}{c}\text { Gas } \\
\text { demand, } \\
\mathrm{G}_{\mathrm{w}, \mathrm{s}, \mathrm{m}^{3}}\end{array}$ \\
\hline 1 & 5 & 55 & 75 & 0.0 & 1.06 & 0 & 5 & 36 & 36 \\
\hline 2 & 5 & 55 & 120 & 0.0 & 1.09 & 0 & 5 & 58 & 58 \\
\hline 3 & 5 & 55 & 180 & 0.0 & 1.16 & 0 & 5 & 87 & 87 \\
\hline 4 & 5 & 55 & 230 & 0.0 & 1.25 & 0 & 5 & 112 & 112 \\
\hline 5 & 4 & 55 & 700 & 0.0 & 1.33 & 0 & 4 & 272 & 272 \\
\hline 6 & 4 & 55 & 500 & 0.0 & 1.41 & 0 & 4 & 194 & 194 \\
\hline 7 & 0 & 55 & 350 & 0.0 & 1.48 & 0 & 0 & 0 & 0 \\
\hline 8 & 0 & 55 & 600 & 0.0 & 1.57 & 0 & 0 & 0 & 0 \\
\hline
\end{tabular}


Table 7. The forecast of annual gas demand for space heating in single-family house.

\begin{tabular}{|c|c|c|c|c|c|c|c|c|c|}
\hline Scope & $\begin{array}{c}\text { Partial } \\
\text { heat load } \\
\mathrm{Q}_{\mathrm{h}, \mathrm{s}} \mathrm{kW}\end{array}$ & $\begin{array}{c}\text { Heating } \\
\text { system } \\
\text { tempera- } \\
\text { ture, }{ }^{\circ} \mathrm{C}\end{array}$ & $\begin{array}{c}\text { Operation } \\
\text { hours, } \\
\mathrm{t}_{\mathrm{t}, \mathrm{s}}, \mathrm{h}\end{array}$ & $\begin{array}{c}\text { Available } \\
\text { GAHP } \\
\text { th.capacity } \\
\mathrm{Q}_{\mathrm{GAHP}, \mathrm{h}, \mathrm{s}} \\
\mathrm{kW}\end{array}$ & $\begin{array}{c}\text { GAHP } \\
\text { in heating } \\
\text { mode }\end{array}$ & $\begin{array}{c}\text { GAHP gas } \\
\text { demand } \\
\mathrm{G}_{\mathrm{GAHP}, \mathrm{h}, \mathrm{s}} \\
\mathrm{m}^{3}\end{array}$ & $\begin{array}{c}\mathrm{HB} \\
\text { thermal } \\
\text { capacity, } \\
\mathrm{QHB}_{\mathrm{HB}, \mathrm{h},} \\
\mathrm{kW}\end{array}$ & $\begin{array}{c}\text { HB gas } \\
\text { demand } \\
\mathrm{G}_{\mathrm{HB}, \mathrm{h}, \mathrm{s},}, \\
\mathrm{m}^{3}\end{array}$ & $\begin{array}{c}\text { Gas } \\
\text { demand, } \\
\mathrm{G}_{\mathrm{h}, \mathrm{s}, \mathrm{s}} \mathrm{m}^{3}\end{array}$ \\
\hline 1 & 22 & 55 & 75 & 12.0 & 1.07 & 147 & 10 & 73 & 220 \\
\hline 2 & 20 & 50 & 120 & 29.5 & 1.20 & 192 & 0 & 0 & 192 \\
\hline 3 & 17 & 45 & 180 & 33.7 & 1.32 & 222 & 0 & 0 & 222 \\
\hline 4 & 14 & 40 & 230 & 38.5 & 1.50 & 205 & 0 & 0 & 205 \\
\hline 5 & 11 & 40 & 700 & 39.6 & 1.60 & 458 & 0 & 0 & 458 \\
\hline 6 & 8 & 35 & 500 & 41.2 & 1.69 & 224 & 0 & 0 & 224 \\
\hline 7 & 5 & 35 & 350 & 41.5 & 1.70 & 96 & 0 & 0 & 96 \\
\hline
\end{tabular}

Annual gas demand for heating: $G_{h}=1617 \mathrm{~m}^{3} / \mathrm{a}$

Table 8. The forecast of annual gas demand for domestic hot water in single-family house.

\begin{tabular}{|c|c|c|c|c|c|c|c|c|c|}
\hline Scope & $\begin{array}{c}\text { DHW } \\
\text { heat load } \\
\mathrm{Q}_{\mathrm{w}, \mathrm{s}}, \mathrm{kW}\end{array}$ & $\begin{array}{c}\text { DHW } \\
\text { system } \\
\text { tempera- } \\
\text { ture, }{ }^{\circ} \mathrm{C}\end{array}$ & $\begin{array}{c}\text { Operation } \\
\text { hours, } \\
\mathrm{t}_{\mathrm{o}, \mathrm{s}}, \mathrm{h}\end{array}$ & $\begin{array}{c}\text { Available } \\
\text { GAHP } \\
\text { th.capacity } \\
\text { Q }_{\text {GAHP,w,s, }} \\
\text { kW }\end{array}$ & $\begin{array}{c}\text { GAHP } \\
\text { GUE } \\
\text { in DHW } \\
\text { mode }\end{array}$ & $\begin{array}{l}\text { GAHP gas } \\
\text { demand } \\
\mathrm{G}_{\mathrm{GAHP}, \mathrm{w}, \mathrm{s}}, \\
\mathrm{m}^{3}\end{array}$ & $\begin{array}{c}\text { HB } \\
\text { thermal } \\
\text { capacity, } \\
\text { QHB,w,s, }_{\text {kW }}\end{array}$ & $\begin{array}{c}\mathrm{HB} \text { gas } \\
\text { demand } \\
\mathrm{G}_{\mathrm{HB}, \mathrm{w}, \mathrm{s}}, \\
\mathrm{m}^{3}\end{array}$ & $\begin{array}{c}\text { Gas } \\
\text { demand, } \\
\mathrm{G}_{\mathrm{w}, \mathrm{s}}, \mathrm{m}^{3}\end{array}$ \\
\hline 1 & 5.5 & 55 & 75 & 26.7 & 1.07 & 37 & 0 & 0 & 37 \\
\hline 2 & 5.5 & 55 & 120 & 27.5 & 1.12 & 56 & 0 & 0 & 56 \\
\hline 3 & 5.5 & 55 & 180 & 29.2 & 1.16 & 81 & 0 & 0 & 81 \\
\hline 4 & 5.5 & 55 & 230 & 31.5 & 1.25 & 96 & 0 & 0 & 96 \\
\hline 5 & 5.5 & 55 & 700 & 33.5 & 1.37 & 268 & 0 & 0 & 268 \\
\hline 6 & 5.5 & 55 & 500 & 35.7 & 1.46 & 179 & 0 & 0 & 179 \\
\hline 7 & 5.5 & 55 & 350 & 37.3 & 1.54 & 119 & 0 & 0 & 119 \\
\hline 8 & 5.5 & 55 & 600 & 40.0 & 1.55 & 203 & 0 & 0 & 203 \\
\hline
\end{tabular}

The gas demand forecasting results and real gas consumption for three tested cases were compared and presented in table 9 and fig. 4. The real gas consumption was measured in at least two years period between 2012 and 2017 in standard operation conditions. The forecasting error is below $10 \%$ in all cases. It is a good result for a simple tool.

Table 9. The forecast and real annual gas consumption in tested cases.

\begin{tabular}{|l|c|c|c|}
\hline \multicolumn{1}{|c|}{ Parameter } & Family Support Center & Primary School & Single-family house \\
\hline Gas demand forecast, $\mathrm{m}^{3} / \mathrm{a}$ & 10388 & 12796 & 2657 \\
\hline Real gas consumption, $\mathrm{m}^{3} / \mathrm{a}$ & 11294 & 11754 & 2729 \\
\hline Forecast error & $-8 \%$ & $+9 \%$ & $-3 \%$ \\
\hline
\end{tabular}



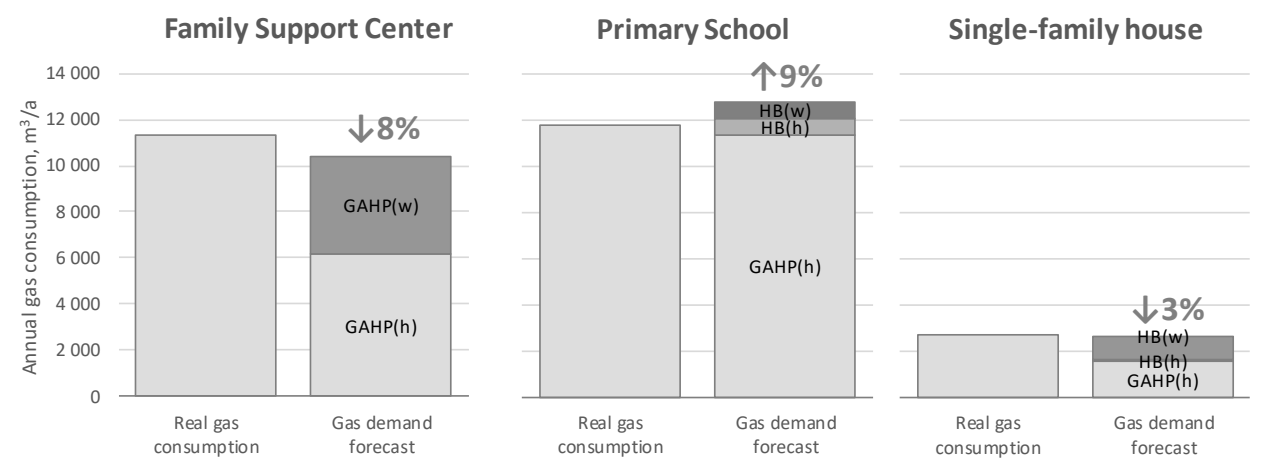

Fig. 4. The real annual gas consumption and Gazuno gas demand forecast in analysed GAHP systems.

\section{Conclusions}

The simple method developed by Gazuno delivers good results in forecasting of annual gas demand compared to real gas consumption data. The three presented cases confirm the correctness of the methodology. Forecasting accuracy within a 3-9\% margin of error is sufficient to support of decision-making at early stage of technical design.

The quick and simple forecasting tool includes technical and operational features of GAHP technology and requires only the basic information about heat demand profile and GAHP system as input data. These advantages encourage to using it for engineering tasks.

The gas demand forecast allows to compare the different GAHP systems with each other and with other heat sources. Obtained by Gazuno methodology results are suitable as the basis for economic calculation of savings, return time or future operation costs. The same calculation scheme can be develop for gas demand forecasting in GAHP cooling or heating and cooling operation mode.

\section{References}

1. M. Aprile, R. Scoccia, T. Toppi, M. Guerra, M. Motta, Int J Refrig, 66, 2016.

2. V. Sharma, B. Shen, C. Keinath, M. Garrabrant, P. Geoghegan, European Regional Climate Zone Modeling of a Commercial Absorption Heat Pump Hot Water Heater, 12th IEA Heat Pump Conference 2017.

3. Gazuno, GAHP A Air-Water gas absorption heat pump, product literature, 2017.

4. Gazuno, Handbook for the K18 range, 2017.

5. W. Wu, B. Wang, W. Shi, X. Li, Appl Energ, 130, 2014

6. X. Li, W. Wu, X. Zhang, W. Shi, B. Wang, Appl Therm Eng, 48, 2012

7. M. Fumagalli, A. Sivieri, M. Aprile, M. Motta, M. Zanchi, Renew Energ, 110, 2017

8. R. Scoccia, T. Toppi, M. Aprile, M. Motta, Journal of Building Engineering, 16, 2018. 